\title{
V.3 Empirische Forschungsfragen
}

Da das zu analysierende Phänomen - nämlich die Etablierung und Vernetzung alternativer Nachrichtenmedien im deutschsprachigen Raum - bisher aus einem eingeschränkten Blickwinkel betrachtet wurde (nämlich dem bereits erörterten Fokus auf rechtskonservative Nachrichtenseiten insbesondere aus dem US-amerikanischen Sprachraum), wurden für dieses Projekt die Forschungsfragen bewusst offen gehalten. Dies spiegelt sich auch gleichzeitig in dem vorwiegend qualitativen Vorgehen wider, das Phänomen vollumfänglich und ohne zu einschränkende Vorannahmen empirisch zu betrachten. Explorative Studien verzichten dabei (in der Regel) auf die Aufstellung von Hypothesen und betrachten das zu untersuchende Phänomen möglichst unvoreingenommen, um keine wesentlichen Aspekte zu übersehen. Unter der Annahme einer Netzwerk- oder Plattformöffentlichkeit auf Social Media und einer dahingehenden Vernetzung alternativer Nachrichtenmedien im deutschsprachigen Raum ergibt sich folgende übergeordnete Forschungsfrage:

FF: Wie lassen sich Gegenöffentlichkeiten wie alternative Online-Nachrichtenmedien in Deutschland, Österreich und der deutschsprachigen Schweiz definitorisch einordnen und welchen Stellenwert nehmen sie in der öffentlichen Kommunikation auf digitalen Plattformen ein?

Aus der klaffenden Forschungslücke, dass alternative Nachrichtenmedien im DACH-Raum noch nicht ausreichend und vor allem unter dem Blickwinkel des digitalen Strukturwandels der Öffentlichkeit definiert wurden, soll in einem ersten Schritt das Feld deutschsprachiger alternativer Online-Medien aufgearbeitet werden. Mittels eines iterativen, induktiven Verfahrens werden zunächst Nachrichten-Websites gesammelt, die sich nach eigenen Angaben in Opposition zur hegemonialen Öffentlichkeit aus Politik und Medien positionieren. Zudem wird deskriptiv aufgezeigt, welche Reichweiten die Seiten in Form von Nutzer_innenzugriffen verzeichnen und welche Social-Media-Plattformen sie mit eigenen Accounts bedienen. Daraus resultiert nachfolgende Forschungsfrage 1, die gleichzeitig den ersten Teil der Untersuchung dieses Projekts leitet:

FF1: Welche alternativen Nachrichtenmedien aus Deutschland, Österreich und der deutschsprachigen Schweiz agieren online mittels eigener Webseite und aufwelchen digitalen Plattformen sind diese vertreten? Welche Reichweiten in Form von Seitenzugriffen respektive Community-Grössen auf Social Media erzielen sie dabei?

Im Sinne einer angestrebten Bestandsaufnahme unterschiedlicher Nachrichtenseiten im Internet ist zudem davon auszugehen, dass aktuell unterschiedliche Formen oder Typen dieser Medien existieren. Vorannahme ist hierbei, dass der 
digitale Strukturwandel der Öffentlichkeit vor allem dadurch geprägt ist, dass sich neben etablierten, klassischen Informationsmedien mit hohen Reichweiten auch neue, disperse Kommunikator_innen etablieren, die zwar im Sinne einer «Longtail-Öffentlichkeit» geringere Reichweiten erzielen, aber den klassischen Informationsjournalismus zunehmend vor Herausforderungen stellen (vgl. Kapitel II.3) (vgl. hierzu auch Eisenegger, 2017; Neuberger, 2009, 2011). Forschungsfrage 2 beschäftigt sich dementsprechend mit Definitionskriterien alternativer Nachrichtenmedien, anhand derer zusätzlich unterschiedliche Typen unterschieden werden können.

FF2: Wie lassen sich alternative Online-Nachrichtenmedien in Deutschland, Österreich und der deutschsprachigen Schweiz anhand des auf ihren Webseiten veröffentlichten Selbstverständnisses typisieren und definieren?

Nachdem im Zuge der ersten beiden Forschungsfragen eine Bestandsaufnahme einschlägiger Medien und deren Kanäle im deutschsprachigen Sprachraum erfolgt und eine Definition und Typisierung dieser vorgenommen wird, widmet sich das Forschungsprojekt anschliessend der Vernetzung der identifizierten Medien auf Social Media. Diesbezüglich wird vorweg angenommen, dass sich vor allem Gegenöffentlichkeiten durch gegenseitige Bezugnahmen etablieren können, indem sie dadurch ihre Definitionsmacht steigern. Schliesslich ist die Vernetzung u. a. als zentrale Logik sozialer Medien zu betrachten (Klinger \& Svensson, 2015; van Dijck \& Poell, 2013). Logiken wie diese ermöglichen es neuen Kommunikator_ innen (wie auch Lai_innen der so genannten Longtail-Öffentlichkeit [Neuberger, 2011]), öffentlich zu kommunizieren und teils sogar beachtliche Reichweiten in Form von z. B. Likes oder Retweets zu erreichen. Zudem wird davon ausgegangen, dass alternative Newssites vor allem länderübergreifend agieren und nicht ausschliesslich länderspezifisch ihre Zielgruppen anzusprechen versuchen (Vogler, 2017). Von Interesse ist dahingehend nicht nur die Vernetzung alternativer Nachrichtenmedien untereinander; auch die Relationen zum professionellen Informationsjournalismus wie auch weiteren öffentlichen Akteur_innen, die eine potenzielle Schlüsselrolle im Netzwerk einnehmen können, wie z. B. Politiker_innen, Wissenschaftler_innen oder weitere Akteur_innen des öffentliches Lebens, sollen untersucht werden. Da die Social-Media-Plattform Twitter besonders für journalistische Zwecke genutzt wird und als eine Art «Elitennetzwerk» aus Journalismus und Politik betrachtet werden kann (Ausserhofer \& Maireder, 2013; Rauchfleisch \& Metag, 2016), eignet sich diese als empirischer Wirklichkeitsausschnitt für dahingehende Analysen. Zudem erweist sich auch aus forschungspraktischen Gründen die Plattform als am geeignetsten für dieses Forschungsprojekt (vgl. Kapitel IV.2.1). Forschungsfrage 3 bezieht sich demzufolge auf die Netzwerkstrukturen alternativer Nachrichtenmedien auf Twitter, aber auch in Relation zu professionellen 
Nachrichtenmedien. Dabei sind die Followerbeziehungen der einschlägigen Medien von Interesse. Durch die Analyse dieser Beziehungsnetzwerke lassen sich unterschiedliche Communities identifizieren, in denen alternative Nachrichtenmedien abonniert werden. Diese Communities können u. a. auch Aufschluss darüber geben, welche Medien- respektive Newsrepertoires sich in bestimmten Communities manifestieren, oder aber auch darüber, ob fragmentierte Teilpublika existieren. Weiter soll anhand von Retweet-Netzwerken eruiert werden, welche Akteur_innen in Form von Retweets auf alternative Nachrichtenmedien Bezug nehmen und vice versa. Für beide Analysen werden Twitterdaten erhoben und analysiert. Somit ist ein deskriptiver Überblick über die Netzwerkstrukturen der Medien aufzeigbar.

FF3: Wie sind alternative Nachrichtenmedien untereinander wie auch mit professionellen Nachrichtenmedien und weiteren Akteur_innen des öffentlichen Lebens auf der Digitalplattform Twitter vernetzt?

Nachdem sich Forschungsfrage 3 vorwiegend auf die strukturelle Ebene dieser Relationen bezieht und einen deskriptiven Überblick verschafft, widmet sich Forschungsfrage 4 der inhaltlichen Perspektive dieser. Im Sinne der relationalen Soziologie wird angenommen, dass ein Netzwerk nicht bloss aus starren Strukturen besteht, sondern aus Sinn bzw. Narrativen zwischen Individuen (Mützel \& Fuhse, 2010, S. 8). Um den Stellenwert alternativer Medien in der öffentlichen Kommunikation zu untersuchen, erscheint es zudem essentiell, besonders Relationen zwischen dem etablierten, reichweitenstarken Journalismus, politischen Entscheidungsträger_innen wie auch weiteren definitionsmächtigen Akteur_innen des öffentlichen Lebens interpretativ genauer zu betrachten. Auch diese Forschungsfrage wird bewusst offengehalten, was dem interpretativen, qualitativen Vorgehen entspricht.

FF4: Inwiefern und nach welchen Logiken nehmen alternative Nachrichtenmedien auf Twitter untereinander, aber auch auf den professionellen Journalismus und Akteur_innen des öffentlichen Lebens wie auch umgekehrt Bezug?

Die vier Forschungsfragen werden methodisch in chronologischer Reihenfolge bearbeitet, die Resultate einzelner Forschungsfragen fliessen zudem in jede weitere empirische Analyse mit ein. Da die Forschungsfragen bewusst offen formuliert wurden und das Vorgehen sehr explorativ ist, wird auf das Aufstellen konkreter Hypothesen und etwaiger Sub-Forschungsfragen verzichtet. Etwaige Vorannahmen oder Thesen, die aus vorhergehenden Analyseschritten resultieren können, werden innerhalb der jeweiligen Kapitel erörtert. Das methodische Vorgehen und die Erhebung der Daten werden im nachfolgenden Kapitel skizziert. 